\title{
Study on Wood Board Defect Detection Based on Artificial Neural Net- work
}

\author{
Lin Wenshu, Shao Lijun and Wu Jinzhuo*
}

\author{
College of Engineering and Technology, Northeast Forestry University, Harbin, Heilongjiang, 150040, P.R. China
}

\begin{abstract}
Due to the increasing contradiction between supply and demand of timber resources, it is necessary to realize the reasonable utilization of the resources. Fast and accurate identification of the location and size of the defects on a piece of wood board is a premise of efficient utilization. In this paper, the identification and positioning of board defects by computer vision and artificial neural network technology was discussed. Through the acquisition of the board image by CCD camera and image processing by using MATLAB, the location and size of the defects on the board was obtained. Finally, the artificial neural network was constructed to identify the defects, and the results showed that board defect identification rate can reach $86.67 \%$. The study has provided a new idea and method to improve lumber recovery, which also provided a theoretical and technical foundation for advanced automation and intelligent wood processing industry.
\end{abstract}

Keywords: Artificial neural network, Computer vision technology, Defect testing, Image processing.

\section{INTRODUCTION}

Timber is one of the indispensable natural materials in daily life. The forest resources in China are relatively poor compared to other countries, so a substantial part of timber consumption has to be met by import and the contradiction between timber demand and supply becomes increasingly prominent. Therefore, it is a radical strategy for relieving the contradiction to protect the existing forest resources, make rational use of the limited resources, tap the potentials of the resources, and improve the processing and utilization rate of timber. Among these measures, strengthening the innovation on timber production technology and improving the processing and utilization rate of wood boards is a very effective way. To achieve this goal, it is necessary to get fast and accurate information on types, locations, and sizes of defects on the wood boards.

Artificial neural network is a kind of information processing system, which processes information through dynamic processes just like the interactions between neurons of human brains. Currently, some studies on the application of artificial neural network in wood board defect detection have been conducted. For example, Jordan et al. found that different wood species had different elastic responses. With further processing, a self-constructed artificial neural network could be used to identify wood species [1]. Jiang et al. successfully related microstructure parameters to physical features of timber through artificial neural networks [2]. Dai et al. applied artificial neural network to extract colors from wood board surface. The results showed that this method could be used to classify the species of wood boards [3].

* Address correspondence to this author at the College of Engineering and Technology, Northeast Forestry University, Harbin, 150040, P.R. China; Tel: 15663790610; E-mail: wujinzhuo1980@163.com
$\mathrm{Xu}$ et al. used artificial neural network to identify the bark and knots on the $\log$ CT images and found that the method can achieve better effect in recognizing larger defects [4]. Qi and Mu employed X-ray to conduct nondestructive testing to extract the characteristic parameters of wood board defects from the established artificial neural network [5]. However, few studies have been conducted to process the wood board image acquired with computer vision technology, so computer vision technology and artificial neural network are used for recognition of wood board defects and position defection in this paper.

\section{ACQUISITION OF EXPERIMENTAL MATERIALS}

The defective wood boards used in this paper were from a sawmill that is located in the outskirts of Harbin, the provincial capital of Heilongjiang province, China. The wood specie was Fraxinus mandschurica Rupr. There are many kinds of wood defects, including knot, insect damage, rot, crack, discoloration, scars, etc., which may have different impacts on the use of wood boards such as reduction of strength and artistic value of wood texture. Among the wood defects, knots are very common. Therefore, the knots on wood boards are taken as the focus of this study. Measurement and statistics are made on the wood board defects, so that comparison and analysis with the result of processed image can be conducted.

\section{IMAGE ACQUISITION AND PROCESSING}

\subsection{Image Acquisition}

A Daheng model DH-HV1351UM USB industrial camera was used to obtain the images of wood boards with knots, insect damage, and cracks. The camera uses progressive ex- 


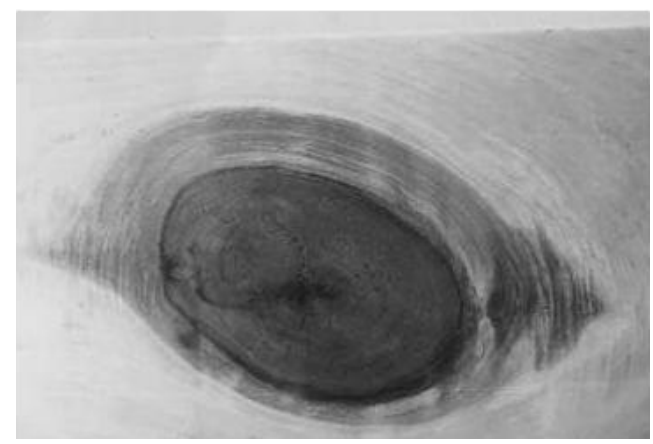

(a)

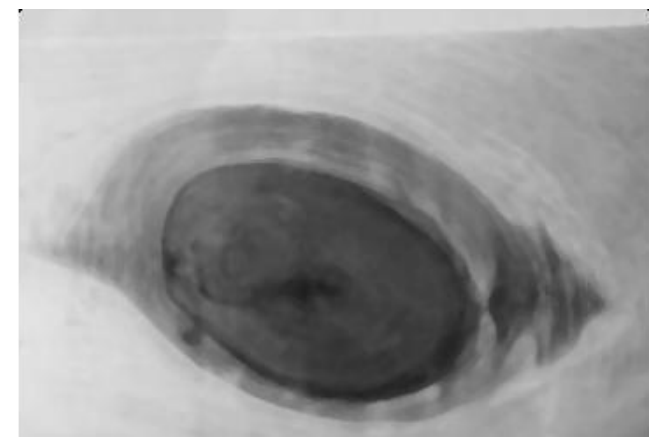

(b)

Fig. (1). The images of grayscale and median filtering.

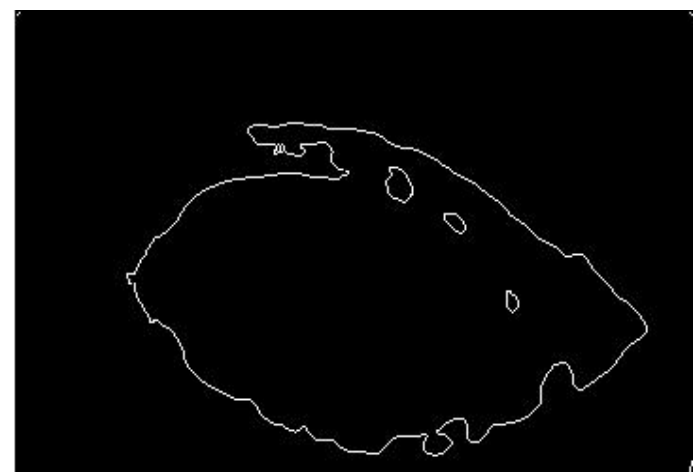

Fig. (2). Edge detection image by Sobel operator.

posure, with programmable exposure time and resolution of 1.3 million pixels. To ensure that the defects on both the upper and lower parts of the wood board can be detected, the CCDs were arranged perpendicularly on both the upper and lower parts of the wood board. The images captured by the camera can be conveniently transferred to a computer through a USB 2.0 port and be saved there.

\subsection{Image Pre-Processing}

Image pre-processing includes noise elimination, image smoothing, and extraction of the edges. Prior to the elimination of noise, the image was first converted to a grayscale image (Fig. 1a). A smoother image can be obtained after noise was removed through filtering operation. The median filtering method was finally selected after effect comparison among several filters. In median filtering, a window that contains an odd number of points was used. All pixels in the adjacent areas were sorted according to their gray scale level based on the method of the theory of scheduling and statistics. At last, all pixels within the window were assigned a median value. Median filtering has good effect in noise inhibition, elimination of isolated noise sources, and smoothing of impulse noise. The method can also retain detailed image information to the maximum extent with noise elimination. The image of median filtering is shown in Fig. (1b).

During edge detection, comparison was made between Canny operator, Prewitt operator, Robert operator, Log operator, and Sobel operator. Sobel operator was finally selected based on the comparison result, since it can not only detect the edge, but also eliminate some noise and the acquired image has few discontinuities. It is especially good in image processing for images with gradual changing grayscale. It also has high detection sensitivity and good effect on diagonal edges. Besides, Sobel operator can form a closed pattern (Fig. 2), which is convenient for subsequent processing 

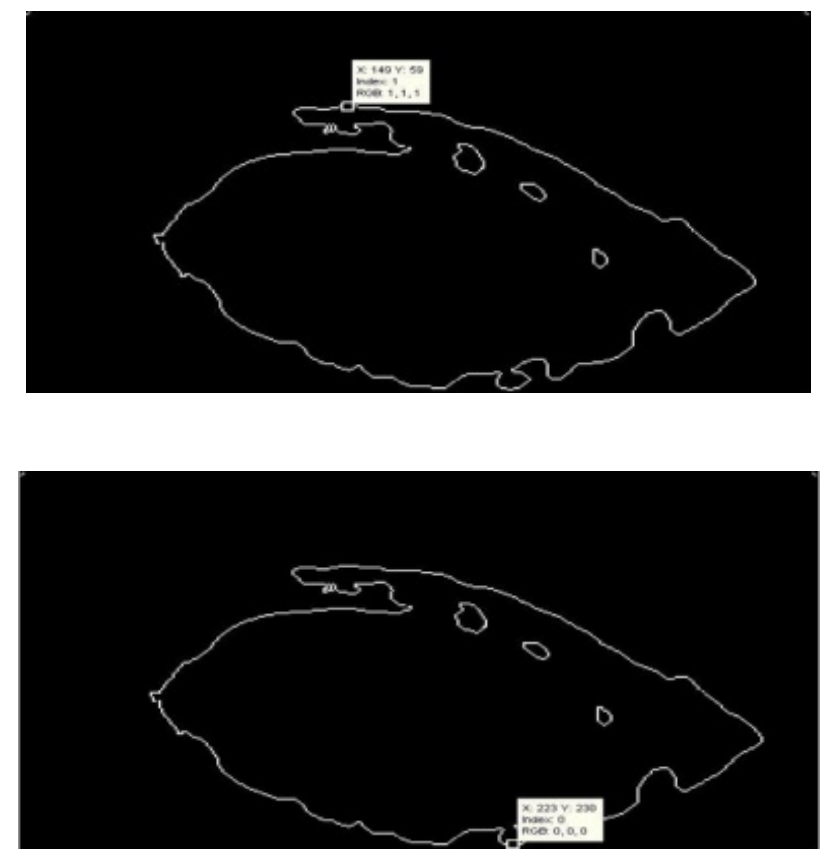

Fig. (3). The images with extreme point coordinates.

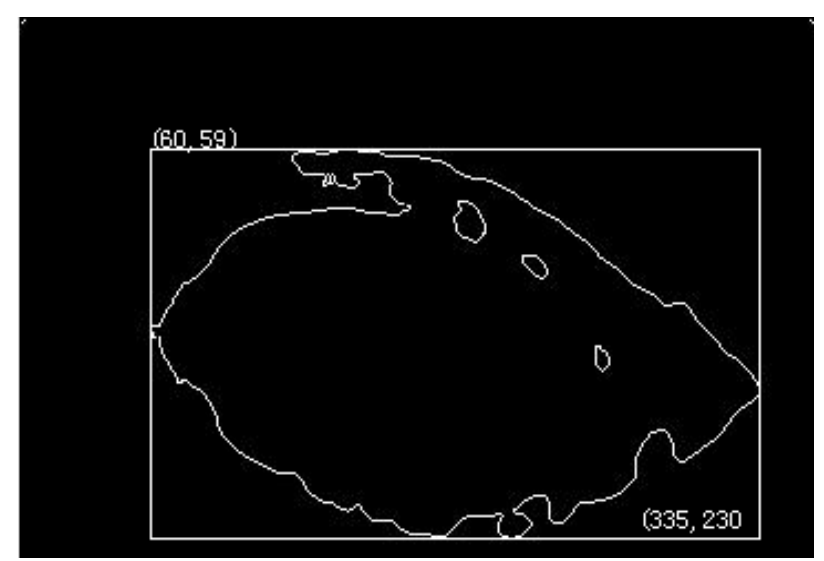

Fig. (4). The image of rectangular area with extreme point coordinates.

\subsection{Interim Image Processing}

The purpose of interim image processing is to find out the coordinate position of the defects. In order to obtain the coordinates of the defect area, it is just necessary to find the coordinates of all black-point pixels in a binary image. In this way, we can get an array of coordinates that contain all pixel points of the defective area. The extreme values of the array can constitute a rectangle. The size of this rectangle is regarded as the size of the knot. The minimum and maximum values of $X$ and $Y$ coordinates of the four points on top, bottom, right and left of the knot are shown in Fig. (3). The location coordinates of the whole knot can be obtained, as shown in Fig. (4). To verify the difference of the obtained rectangular area and the original defect size, the obtained rectangular area was compared with the rectangular area formed by the original defect. The result showed that the processed area accounted for $91 \%$ of the original area, which basically met the requirement for processing.

\subsection{Image Post-Processing}

The work of image post-processing is to cut out the defect target area, to separate and highlight the defect (knot, insect damage, and crack) area. Meanwhile, the characteristic quantities of the region were extracted, which were used as the inputs to the artificial neural network. When separating the defect area, in consideration of the special features of the binary image, the grayscale image and the image after edge extraction were superimposed. This operation can eliminate the background of the grayscale image. As a result, only the defective part of the image has grayscale value and other area is of white color. In this way, the characteristic array for the defect grayscale values was obtained. The superimposed image is shown in Fig. (5).

How to select the characteristic quantities? When we carry out mode recognition to a defective wood board, we need to have values that can describe the defect characteristics. It was found that through processing of all kinds of defects, 


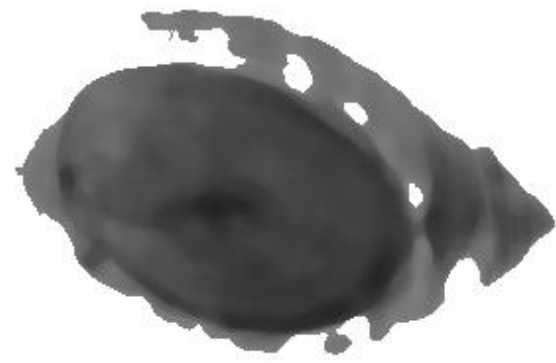

Fig. (5). Image addition by the gray image and image after edge extraction.

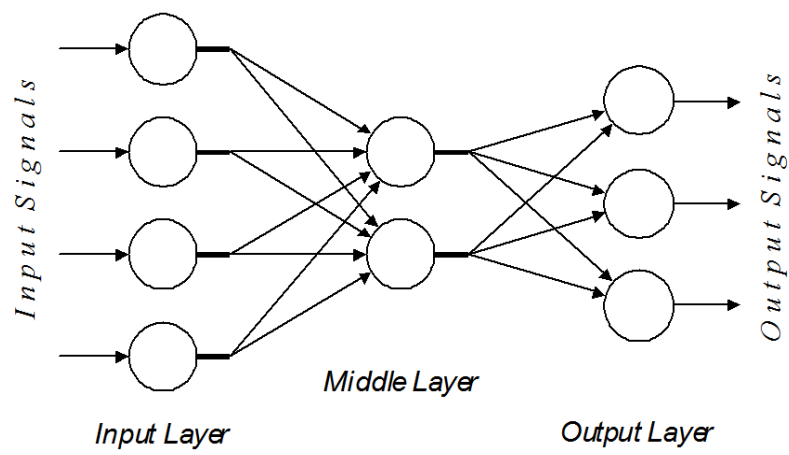

Fig. (6). Artificial neural network model.

different defects had significantly different grayscale levels. Knots had gradually changing grayscale level. Insect damage was represented by the same level of grayscale. Cracks were represented by a wide span of grayscale level. Therefore, grayscale can be chosen as a characteristic quantity. In addition, the length-width ratios are different for different kinds of defects. For example, knots are usually in round shape, rot in more or less irregular shape and cracks in strip. These characteristics can be used to describe different defect features of the wood board. So the mean grayscale of the defect area, grayscale variance, and length-width ratio were selected as the characteristic quantities of the defects.

\section{MODE RECOGNITION BY ARTIFICIAL NEURAL NETWORK}

\subsection{Construction of Artificial Neural Network}

Learning (also known as training) of artificial neural network is the core of artificial neural network [6]. Samples are continuously input to the network in the learning process. The connection weights of various layers of the network are changed in accordance with the error. The change of weights is realized through a network transfer function. In this way making the output of the network gradually approaches the desired output. Construction of artificial neural network can promote defect recognition and improve the utilization of defective wood board.
BP neural network was chosen in this paper. Wood defect mode recognition was achieved based on the principle that different types of defects have different characteristic values. BP algorithm is a learning algorithm of multilayer propagation network, also known as error back propagation learning algorithm. The basic idea of BP algorithm is that during learning process, signals propagate forward from the front to the back. After forward propagation, an error to the expectation is output. Now, to make the network more accurate, back propagation of error is needed. Error is transferred to the input layer for weight adjustment. Finally an error-free adaptive result can be achieved. An artificial neural network model is shown in Fig. (6).

To construct an artificial neural network, it is necessary to input the number of nodes in the input layer, the number of hidden layers, the number of nodes in the hidden layer, sample recognition types as well as the error precision.

(1) Number of nodes in the input layer. With regard to the above-mentioned extraction of characteristic quantities, there are three characteristic quantities, including the lengthwidth ratio of defect, grayscale, and variance of defect. So, the number of nodes in the input layer was 3 .

(2) Number of hidden layers. With reference to various literatures and tests, it is found that one hidden layer is enough to process defect recognition problem in this paper. When the number of hidden layers increases, the training time will increase dramatically. With the consideration of 


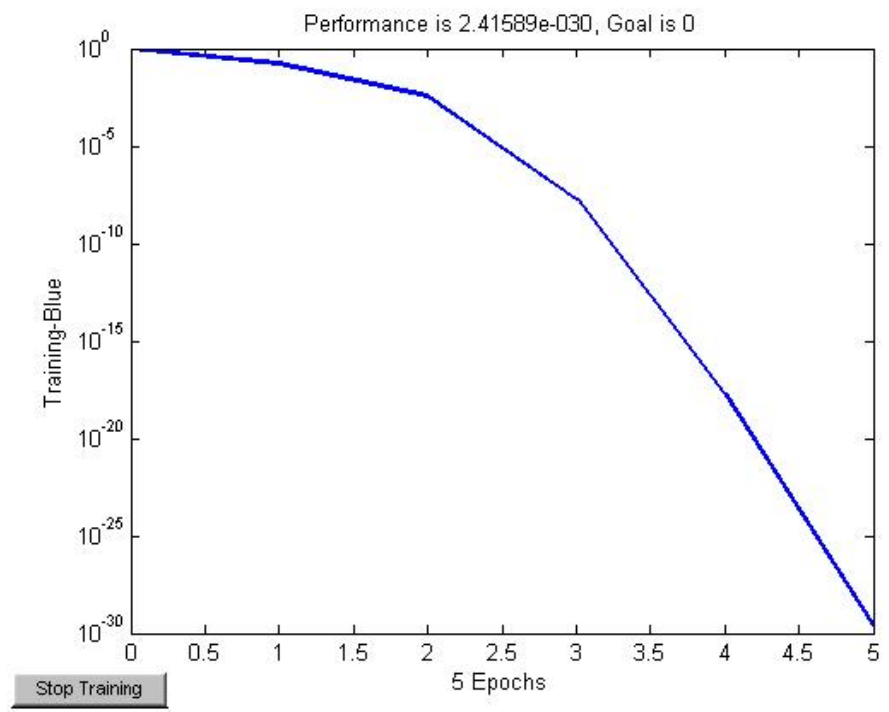

Fig. (7). Training results.

getting accurate and clear results, one hidden layer was adopted in this paper.

(3) Number of nodes in the hidden layer. The effect of the entire network depends on the number of nodes in the hidden layer. If the chosen number is too high, the effect of sample noise will expand, resulting in inaccurate network recognition and overtraining. If the chosen number of nodes in the hidden layer is too low, the network training may become inaccurate, and when a complicated problem is processed, the method may fail to meet the requirement. When determining the number of nodes in the hidden layer, the empirical formula was used:

$S=\sqrt{R+K}+\alpha$

Where,

$\mathrm{S}$ is the number of nodes in the hidden layer,

$\mathrm{R}$ is the number of input nodes,

$\mathrm{K}$ is the number of output nodes, and

$\alpha$ is a constant between 1 and 10 .

(4) Number of sample recognition types. Since the study focuses on recognition of knots, insect damage, and crack, the number of sample recognition modes was set to 3 .

(5) Error accuracy. The initial error accuracy was chosen to be 0.001 . It may be changed after testing. If it is difficult to achieve convergence, it can be changed to 0.01 , otherwise the error accuracy can be changed to 0.0001 or 0.00001 . To improve the error accuracy, it is necessary to increase the number of nodes in the hidden layer. Higher accuracy is usually obtained on the expense of more training time.

\subsection{Network Training and Results}

(1) Training results.

Fig. (7) shows the results of artificial neural network training. The training results showed that the network can easily meet the required error standard and the rate of learning was satisfactory, which indicated that the chosen number of nodes in the hidden layer of network was correct. So, excessive learning can be avoided and good effect can be expected.

\section{(2) Results of recognition}

There were a total of 150 samples in the test, including 50 knots, 50 cracks, and 50 insect pests. Forty-five samples of each type were selected as the training data for artificial neural network, and the remaining five were used to test the recognition rate of the artificial neural network. For the convenience of easy recognition, the output value was regarded as 0 if its absolute value was less than 0.4 . The output value was regarded as 1 if its absolute value was greater than 0.7 . When the absolute value of output value fell within $[0.4$, $0.7]$, it was regarded as 2 . The result indicated that the average recognition rate of the artificial neural network can reach $86.67 \%$. The effect of recognition was the best for insect pests, as high as $100 \%$. The specific recognition rate for each sample is shown in Table $\mathbf{1 .}$

\section{CONCLUSION}

In this paper, a CCD camera was used to take the image of defective wood board. The image then underwent smoothing and filtering processing by software MATLAB. The defect image was further processed by median filtering and Sobel operator, to highlight the defect image for easier processing. Three characteristic quantities, including the lengthwidth ratio, grayscale, and variance of defects, were extracted from the processed image. At last the self-constructed artificial neural network was established to recognize the wood board defects. The recognition rate can reach $86.67 \%$. This study has introduced advanced methods and technologies to timber processing industry and laid a foundation for development of intellectualization and automation in timber processing and production as well as improvement of wood board quality. 
Table 1. The statistics of the defect recognition rate.

\begin{tabular}{|c|c|c|c|c|}
\hline Defect Types & Learning Results & Testing Results & Target Value & Recognition Rate \\
\hline \multirow{5}{*}{ Knots } & {$\left[\begin{array}{lll}0.7927 & 0.1533 & 0.2366\end{array}\right]$} & {$\left[\begin{array}{lll}1 & 0 & 0\end{array}\right]$} & {$\left[\begin{array}{lll}1 & 0 & 0\end{array}\right]$} & \multirow{5}{*}{$80 \%$} \\
\hline & {$\left[\begin{array}{llll}0.8129 & 0.2326 & 0.3205\end{array}\right]$} & {$\left[\begin{array}{lll}1 & 0 & 0\end{array}\right]$} & {$\left[\begin{array}{lll}1 & 0 & 0\end{array}\right]$} & \\
\hline & {$\left[\begin{array}{llll}0.7596 & 0.0565 & 0.2013\end{array}\right]$} & {$\left[\begin{array}{lll}1 & 0 & 0\end{array}\right]$} & {$\left[\begin{array}{lll}1 & 0 & 0\end{array}\right]$} & \\
\hline & {$\left[\begin{array}{llll}0.9571 & 0.4364 & 0.3252\end{array}\right]$} & {$\left[\begin{array}{lll}1 & 2 & 0\end{array}\right]$} & {$\left[\begin{array}{lll}1 & 0 & 0\end{array}\right]$} & \\
\hline & {$\left[\begin{array}{llll}0.9416 & 0.3015 & 0.1032\end{array}\right]$} & {$\left[\begin{array}{lll}1 & 0 & 0\end{array}\right]$} & {$\left[\begin{array}{lll}1 & 0 & 0\end{array}\right]$} & \\
\hline \multirow{5}{*}{ Insect pests } & {$\left[\begin{array}{llll}0.1342 & 0.8516 & 0.0821\end{array}\right]$} & {$\left[\begin{array}{lll}0 & 1 & 0\end{array}\right]$} & {$\left[\begin{array}{lll}0 & 1 & 0\end{array}\right]$} & \multirow{5}{*}{$100 \%$} \\
\hline & {$\left[\begin{array}{lll}0.2633 & 0.7561 & 0.3124\end{array}\right]$} & {$\left[\begin{array}{lll}0 & 1 & 0\end{array}\right]$} & {$\left[\begin{array}{lll}0 & 1 & 0\end{array}\right]$} & \\
\hline & {$\left[\begin{array}{llll}0.0916 & 0.8325 & 0.1645\end{array}\right]$} & {$\left[\begin{array}{lll}0 & 1 & 0\end{array}\right]$} & {$\left[\begin{array}{lll}0 & 1 & 0\end{array}\right]$} & \\
\hline & {$\left[\begin{array}{llll}0.3542 & 0.9187 & 0.2023\end{array}\right]$} & {$\left[\begin{array}{lll}0 & 1 & 0\end{array}\right]$} & {$\left[\begin{array}{lll}0 & 1 & 0\end{array}\right]$} & \\
\hline & {$\left[\begin{array}{llll}0.1513 & 0.8403 & 0.1025\end{array}\right]$} & {$\left[\begin{array}{lll}0 & 1 & 0\end{array}\right]$} & {$\left[\begin{array}{lll}0 & 1 & 0\end{array}\right]$} & \\
\hline \multirow{5}{*}{ Cracks } & {$\left[\begin{array}{llll}0.1069 & 0.2038 & 0.9048\end{array}\right]$} & {$\left[\begin{array}{lll}0 & 0 & 1\end{array}\right]$} & {$\left[\begin{array}{lll}0 & 0 & 1\end{array}\right]$} & \multirow{5}{*}{$80 \%$} \\
\hline & {$\left[\begin{array}{lll}0.4021 & 0.1328 & 0.8459\end{array}\right]$} & {$\left[\begin{array}{lll}2 & 0 & 1\end{array}\right]$} & {$\left[\begin{array}{lll}0 & 0 & 1\end{array}\right]$} & \\
\hline & {$\left[\begin{array}{lll}0.1348 & 0.3124 & 0.7519\end{array}\right]$} & {$\left[\begin{array}{lll}0 & 0 & 1\end{array}\right]$} & {$\left[\begin{array}{lll}0 & 0 & 1\end{array}\right]$} & \\
\hline & {$\left[\begin{array}{llll}0.3042 & 0.2901 & 0.8740\end{array}\right]$} & {$\left[\begin{array}{lll}0 & 0 & 1\end{array}\right]$} & {$\left[\begin{array}{lll}0 & 0 & 1\end{array}\right]$} & \\
\hline & {$\left[\begin{array}{llll}0.3018 & 0.1693 & 0.7983\end{array}\right]$} & {$\left[\begin{array}{lll}0 & 0 & 1\end{array}\right]$} & {$\left[\begin{array}{lll}0 & 0 & 1\end{array}\right]$} & \\
\hline
\end{tabular}

\section{CONFLICT OF INTEREST}

The authors confirm that this article content has no conflict of interest.

\section{ACKNOWLEDGEMENTS}

The authors would like to acknowledge the support of the Fundamental Research Funds for the Central Universities (DL13BB19) and the Scientific Activities Support Program of Chinese State Forestry Administration for Returned Talents from Overseas (415003).

\section{REFERENCES}

[1] R. Jordan, F. Feeney, and N. Nesbitt, "Classification of wood species by natural network analysis of ultrasonic signals", Ultrasonics, vol. 36, pp. 219-222, 1998.
[2] Z.H. Jiang, X.M. Jiang, Y.C. Zhou, Y. An, L. Zhao, and Y.W. Jing, "A kind of NN modeling method of relational model of Chinese for microstructure and its material characteristic", Scientla Silvae Sinicae, vol. 41, pp. 133-139, 2005.

[3] T.H. Dai, K.Q. Wang, X.B. Bai, L.J. Chen, and Y.H. Xie, "Analysis of wood classification based on neural network and color features", Forest Engineering, vol. 22, pp. 18-20, 2006.

[4] Z.J. Xu, H. L. Wang, J.W. Ding, and N. Ye, "Wood defect recognition in $\log$ CT images by artificial neural network", China Wood Industry, vol. 19, pp. 15-17, 2005

[5] D.W. Qi, and H.B. Mu, "Application of artificial neural networks in the testing of wood defects", Forest Engineering, vol. 22, pp. 2123, 2006.

[6] L.H. Wang, H.D. Xu, T. Xing, and S.Y. Ni, "Quantitatively determining of hole-defects in Korean pine lumber based on modal analysis and BP neural network", Scientla Silvae Sinicae, vol. 46, pp. $177-181,2010$

Revised: January 04, 2015

Accepted: February 25, 2015

(C) Wenshu et al.; Licensee Bentham Open.

This is an open access article licensed under the terms of the Creative Commons Attribution Non-Commercial License (http:/creativecommons.org/licenses/bync/4.0/) which permits unrestricted, non-commercial use, distribution and reproduction in any medium, provided the work is properly cited. 